\title{
Mobility Tracking Based on Autoregressive Models
}

\author{
Zainab R. Zaidi, Member, IEEE, and Brian L. Mark, Senior Member, IEEE
}

\begin{abstract}
We propose an integrated scheme for tracking the mobility of a user based on autoregressive models that accurately capture the characteristics of realistic user movements in wireless networks. The mobility parameters are obtained from training data by computing Minimum Mean Squared Error (MMSE) estimates. Estimation of the mobility state, which incorporates the position, velocity, and acceleration of the mobile station, is accomplished via an extended Kalman filter using signal measurements from the wireless network. By combining mobility parameter and state estimation in an integrated framework, we obtain an efficient and accurate real-time mobility tracking scheme that can be applied in a variety of wireless networking applications. We consider two variants of an autoregressive mobility model in our study and validate the proposed mobility tracking scheme using mobile trajectories collected from drive test data. Our simulation results validate the accuracy of the proposed tracking scheme even when only a small number of data samples is available for initial training.
\end{abstract}

Index Terms-Mobility model, geolocation, autoregressive model, Kalman filter, Yule-Walker equations.

\section{INTRODUCTION}

$\mathrm{U}$ SER mobility is a fundamental characteristic of wireless mobile networks that profoundly impacts network performance. To perform optimally, a wireless network should be designed to take into account the mobility of the user. In this regard, two issues of fundamental importance are: 1) the development of suitable models of user mobility to drive realistic simulations of wireless networks and 2) efficient real-time tracking of user mobility to enable seamless connectivity and quality of service in a wireless network. The two issues are closely interrelated, since accurate real-time tracking of user mobility must be based on an appropriate mobility model that can be used to anticipate the future mobility state of the user. Conversely, in order to generate realistic mobility patterns for the purpose of simulating a wireless network, actual mobile trajectories from live networks should be fit to a model that can capture the salient characteristics of user mobility. Furthermore, accurate mobility tracking requires that the parameters of the mobility model be matched as closely as possible to the available data.

Some of the more prominent mobility models (cf. [1], [2]) that have been proposed in the literature include random walk models [3], the random waypoint model [4], Brownian motion models [5], Gauss-Markov models [6], and Markov chain models [7]. Such models have the important feature of simplicity, making them amenable for use in simulation and in some cases analytical modeling of wireless network

- Z.R. Zaidi is with the Networked Systems Group, NICTA, Locked Bag 9013, Alexandria NSW 1435, Australia.

E-mail: Zainab.Zaidi@nicta.com.au.

- B.L. Mark is with the Department of Electrical and Computer Engineering, George Mason University, 4400 University Drive, Fairfax, VA 22030.

E-mail: bmark@gmu.edu.

Manuscript received 10 Feb. 2009; revised 25 Aug. 2009; accepted 6 Jan. 2010; published online 30 June 2010.

For information on obtaining reprints of this article, please send e-mail to: tmc@computer.org, and reference IEEECS Log Number TMC-2009-02-0048. Digital Object Identifier no. 10.1109/TMC.2010.130. behavior. However, more recent studies have shown that many of them do not accurately represent actual user trajectories in real wireless networks [2], [8], [9], [10]. Consequently, such models may provide misleading characterizations of network performance.

Another class of mobility models employs additional information to improve the accuracy of mobility representation. The additional information may consists of a terrain map or street layout [11], [12], traffic conditions [13], hotspots or regions of interest [14], [15], obstacle's shapes and locations [16], behavioral rules to represent typical human responses [17], etc. However, the applicability of such models may be limited since the parameters for one scenario may not be usable in other environments. Since the modeling assumptions are not validated with real data and compared with other models, the accuracy achieved by this class of models is difficult to evaluate quantitatively. Moreover, such models are not sufficiently rich to enable accurate and precise real-time mobility tracking.

A linear system model of mobility has been applied to real-time mobility tracking via various state estimation methods, such as Kalman filters [18], [19], [20], sequential Monte Carlo filtering [21], particle filters [22], and predictive methods [6]. In this model, the mobility state consists of position, velocity, and acceleration. The linear system model is capable of capturing realistic user mobility patterns, but specification of an optimal set of model parameters is not straightforward. Mobility tracking schemes derived from the linear system model are accurate as long as the model parameters match the mobility characteristics of the user. However, accuracy of the model parameters is difficult to achieve in practice.

In this paper, we study two models of mobility based on autoregressive (AR) models, which are amenable to parameter estimation. The first is a sampled version of an underlying continuous-time, first-order AR model. We refer to this model as the AR-1 model. In the AR-1 model, the mobility state consists of the position, velocity, and 
acceleration of the mobile at a given time instant. The AR-1 model was first introduced in [23]. A more comprehensive study, including comparative analysis with other mobility models, convergence issues, effects of training data, and efficacy of the AR-1 model in representing a broader range of trajectories, is given in the present paper.

The AR-1 model is a variant of the linear system model with the key feature that it is amenable to Minimum Mean Squared Error (MMSE) estimation of the model parameters. Optimal parameter estimation is generally not possible with the linear system model of mobility used in [18], [19], [21]. The AR-1 model is sufficiently simple to enable real-time mobility tracking, but general enough to accurately capture the characteristics of realistic mobility patterns in wireless networks by means of optimal estimation of model parameters.

The second AR-based model, which we call the PositionAR model, is a discrete-time model in which the mobility state consists of the mobile's position at consecutive time points. In the Position-AR model, velocity and acceleration are represented as finite differences of position coordinates. As we shall discuss in this paper, parameter and state estimation algorithms for the Position-AR model have smaller computational complexity than those for the AR-1 model. Our numerical results also show that the Position-AR model requires less training data for parameter estimation.

Based on the AR-1 and Position-AR mobility models, we develop a mobility tracking scheme that integrates MMSE estimation for the unknown model parameters with mobility state estimation using Kalman filtering. The mobility tracking scheme can adapt to changes in the mobility characteristics over time, since the model parameters are continuously reestimated using new observation data. Our numerical results using drive test data show that the AR-1 and Position-AR models can accurately capture realistic mobility patterns. Without a systematic approach to parameter estimation, other mobility models cannot make a similar claim.

The main contribution of this paper is a mobility tracking scheme that simultaneously estimates both the mobility state of a mobile user and the unknown parameters of the mobility model, in this case, the AR-1 and Position-AR models. As discussed earlier, other models of mobility proposed in the literature are not amenable to parameter estimation and hence cannot be used, in practice, to accurately model real mobility traces. Our numerical results show that the proposed mobility tracking algorithm, based on either the AR-1 or the Position-AR model, converges quickly and yields accurate performance when a sufficient amount of training data is provided for initialization. The algorithm is computationally feasible for real-time tracking applications, as it requires a small number of Kalman filtering and MMSE estimation steps to be performed at each discrete-time instant. Comparison between the AR-1 and Position-AR models shows that the Position-AR model yields a mobility tracking scheme that has smaller computational complexity and is more robust to variations in operational settings such as the number of available training samples and the distance between mobile nodes and base stations.
The remainder of the paper is organized as follows: Section 2 describes the AR-1 and Position-AR models. Procedures for optimal estimation of the model parameters in the MMSE sense are developed in Section 3. The parameter estimation procedure is one component of an integrated scheme for real-time mobility tracking. Section 4 discusses the second component of mobility state estimation via Kalman filtering using various types of signal measurements from the wireless network as observation data. Section 5 presents a detailed validation of the AR-1 and Position-AR mobility models and their associated mobility tracking schemes using drive test data. Finally, Section 6 concludes the paper.

\section{Autoregressive Mobility Models}

Autoregressive models have been used to model mobility in wireless networks, such as the Gauss-Markov model [6], the position and velocity model [20], etc. The Global Positioning System (GPS) uses a variety of autoregressive-based models, including one called the position, velocity, and acceleration (PVA) model [24]. However, the issue of how to select the appropriate model parameters to represent realistic mobility patterns has not been treated for these models, nor in the available literature on mobility modeling for wireless networks (cf. [1], [2]). In this section, we propose two variants of autoregressive models to represent user mobility in a wireless network. Parameter estimation for these models is discussed in Section 3.

\subsection{AR-1 Model}

The AR-1 model is a discrete-time model of mobility such that the mobility state at time index $n$ is given by $s_{1, n}=\left[x_{n}, \dot{x}_{n}, \ddot{x}_{n}, y_{n}, \dot{y}_{n}, \ddot{y}_{n}\right]^{\prime}$, where $x_{n}$ and $y_{n}$ denote the $x$ and $y$ coordinates, $\dot{x}_{n}$ and $\dot{y}_{n}$ represent the velocity, and $\ddot{x}_{n}$ and $\ddot{y}_{n}$ represent the acceleration of a mobile unit at discrete-time instant $n$ in two-dimensional space. The notation ' indicates the matrix transpose operator. If mobility state information is needed in three dimensions, the $s_{1, n}$ vector can be augmented by $\left[z_{n}, \dot{z}_{n}, \ddot{z}_{n}\right]^{\prime}$, where the vector elements represent position, velocity, and acceleration in the $z$ dimension. We characterize the dynamics of the mobility state process $\left\{s_{1, n}\right\}$ by a first-order autoregressive (AR-1) model given as follows:

$$
s_{1, n+1}=A_{1} s_{1, n}+w_{1, n},
$$

where $A_{1}$ is a $6 \times 6$ transformation matrix and $w_{1, n}$ is a zero mean, white Gaussian vector process with covariance matrix $Q_{1}$. The matrices $A_{1}$ and $Q_{1}$ can be estimated from the trajectory data using Yule-Walker equations as will be discussed in Section 3.

\subsection{Position-AR Model}

Position-AR is also a discrete-time model of mobility but the mobility state at time index $n$ is given by $s_{2, n}=\left[x_{n}, x_{n-1}\right.$, $\left.x_{n-2}, y_{n}, y_{n-1}, y_{n-2}\right]^{\prime}$, where $x_{n}$ and $y_{n}$ denote the $x$ and $y$ coordinates of the mobile unit at discrete-time index $n$. The dynamics equation of the Position-AR model is given by

$$
s_{2, n+1}=A_{2} s_{2, n}+w_{2, n},
$$


where $A_{2}$ is a $6 \times 6$ transformation matrix and $\boldsymbol{w}_{2, n}$ is a zero mean, white Gaussian vector process with covariance matrix $Q_{2}$.

The elements of the matrix $A_{2}$ specify the relationships among position, velocity, and acceleration from time $n T$ to time $(n+1) T$, where $T$ is the time interval between successive samples. In the $x$-direction,

$$
x_{n+1}=x_{n}+T \dot{x}_{n}+\frac{T^{2}}{2} \ddot{x}_{n} .
$$

Using finite differences, $\dot{x}_{n}$ and $\ddot{x}_{n}$ can be approximated as follows:

$$
\begin{aligned}
& \dot{x}_{n}=\frac{x_{n}-x_{n-1}}{T}, \\
& \ddot{x}_{n}=\frac{\dot{x}_{n}-\dot{x}_{n-1}}{T}=\frac{x_{n}-2 x_{n-1}+x_{n-2}}{T^{2}} .
\end{aligned}
$$

Substituting (4) and (5) into (3), we obtain

$$
x_{n+1}=2.5 x_{n}-2 x_{n-1}+0.5 x_{n-2} .
$$

A set of equations analogous to (6) can be written to characterize the system dynamics in the $y$-direction. Hence, the matrix $A_{2}$ is given as

$$
A_{2}=\left[\begin{array}{cc}
A_{x} & 0_{3 \times 3} \\
0_{3 \times 3} & A_{y}
\end{array}\right],
$$

where $0_{3 \times 3}$ is the $3 \times 3$ matrix of all zeros and

$$
A_{x}=A_{y}=\left[\begin{array}{ccc}
2.5 & -2 & 0.5 \\
1 & 0 & 0 \\
0 & 1 & 0
\end{array}\right]
$$

Since $A_{2}$ is a constant matrix, as opposed to the unknown $A_{1}$ matrix of the AR-1 model, estimation of the covariance matrix $Q_{2}$ completely specifies the Position-AR mobility model.

\subsection{Comparison with Other Mobility Models}

Comparing the state equation (1) with that of the linear system model discussed in [18], the linear system model includes an extra term $B \boldsymbol{u}_{n}$, where $B$ is a $6 \times 2$ matrix and $\boldsymbol{u}_{n}$ is a vector of two independent semi-Markov discrete command processes that drive the acceleration of the model in the two-dimensional plane. In the linear system model of [18] and [19], the matrices $A$ and $B$ are specified in terms of the sampling interval $T$ and a parameter $\alpha$. The command process $u_{n}$ is specified by a set of discrete command levels, a transition probability matrix, and probability distributions for the durations in each command level. An outstanding issue for the linear system model is the question of how to specify an appropriate set of parameters.

The Position-AR and AR-1 models are more general than the Gauss-Markov model proposed in [6]. In the GaussMarkov model of [6], the mobility state consists of position, velocity, and direction, but does not explicitly represent acceleration. A key feature of the Gauss-Markov model with respect to simpler mobility models is that the correlation between successive velocity states is explicitly modeled via a gain parameter $\alpha$. A similar model was used in [20] as the basis for a location-tracking scheme. The AR-1 model captures not only the correlation between velocity states, but also the correlation between acceleration states. Similarly, the Position-AR model incorporates the last three successive position coordinates in order to represent the current position so that the effect of velocity, as well as acceleration is incorporated into the model.

A significant benefit of the Position-AR and AR-1 models is that they can be used to provide predictive mobility information. If the state information or estimate $\hat{\boldsymbol{s}}_{n}$ at a given time $n$ is available, it is possible to predict the mobility state at any time $n+m$ in the future. From the theory of autoregressive processes (cf. [25]), the optimal predicted state $s_{n+m \mid n}^{*}$ of a mobile node in the MMSE sense, given the state estimate $\hat{s}_{n}$ at time $n$, can be obtained as

$$
\boldsymbol{s}_{n+m \mid n}^{*}=E\left[\boldsymbol{s}_{n+m} \mid \hat{\boldsymbol{s}}_{n}\right]=A^{m} \hat{\boldsymbol{s}}_{n},
$$

where $A$ could be $A_{1}$ or $A_{2}$ and the mobility state $\hat{\boldsymbol{s}}_{n}$ could be $\hat{s}_{1, n}$ or $\hat{s}_{2, n}$, depending on which of the two mobility models is used. The associated covariance matrix for the predicted mobility state at time $n+m$, denoted $M_{n+m \mid n}^{*}=$ $\operatorname{Cov}\left[s_{n+m}^{*} \mid \hat{\boldsymbol{s}}_{n}\right]$, is given by

$$
M_{n+m \mid n}^{*}=A^{m} M_{n} A^{\prime m}+\sum_{l=0}^{m-1} A^{m-1-l} Q A^{\prime m-1-l},
$$

where $M_{n}=\operatorname{Cov}\left[\hat{\boldsymbol{s}}_{n}\right]$ and $Q$ could be $Q_{1}$ or $Q_{2}$. Knowledge of the predicted mobility state can be used to devise anticipatory resource allocation schemes for wireless networks. For example, the predicted mobility state could be incorporated into IP mobility management protocols [26], [27] to provide more seamless handoffs (cf. [28]). Existing geolocation systems generally track only the current location of the mobile and do not provide this predictive capability.

According to Bettstetter's nomenclature for mobility models [1], the AR-1 and Position-AR models maybe classified as microscopic mobility models. A microscopic model describes the movement, i.e., position, velocity, etc., of an individual vehicle or person as opposed to a model describing group behavior such as the fluid flow model, group mobility model [29], gravity models [30], map or activity-based models [31], [32], etc. Such composite mobility models are constructed from microscopic models (cf. [2]). Thus, the AR-1 and Position-AR models could be used as building blocks to develop more sophisticated models of mobility for various network scenarios.

\section{Mobility Parameter Estimation}

In this section, we discuss algorithms for obtaining the MMSE estimates of the parameters for the AR-1 and Position-AR models based on training data. Using the parameter estimates, the AR- 1 and Position-AR models can be used to generate realistic mobility patterns for simulation purposes, given a suitable set of training samples obtained from the field.

\subsection{AR-1 Parameter Estimation}

Using the Yule-Walker equations [25], an optimal estimate of $A_{1}$ in the MMSE sense, denoted $\hat{A}_{1}^{(n)}$, where $n$ specifies the amount of training data available, can be found from the mobility state data $s_{1,1}, \ldots, s_{1, n}$ as follows:

$$
\hat{A}_{1}^{(n)}=R_{s}^{(n)}(1) R_{s}^{(n)}(0)^{-1}
$$


where

$$
\begin{aligned}
& R_{s}^{(n)}(1)=\frac{1}{n-2} \sum_{i=1}^{n-1} s_{1, i} s_{1, i+1}^{\prime}, \\
& R_{s}^{(n)}(0)=\frac{1}{n-1} \sum_{i=1}^{n} s_{1, i} s_{1, i}^{\prime} .
\end{aligned}
$$

The estimator $\hat{A}_{1}^{(n)}$ is an MMSE estimator and can be directly derived from the orthogonality principle. The noise covariance matrix $\hat{Q}_{1}^{(n)}$ is estimated using the residual estimation error, $e_{i} \triangleq s_{1, i}-\hat{A}_{1}^{(i)} s_{1, i-1}$, as follows:

$$
\hat{Q}_{1}^{(n)}=\frac{1}{n-1} \sum_{i=1}^{n} \boldsymbol{e}_{i} \boldsymbol{e}_{i}^{\prime} .
$$

An extended Kalman filter, described in Section 4.2, is used to generate the mobility state estimates $\hat{s}_{1, n}$ from the wireless measurements $\boldsymbol{o}_{n}$ at time $n$. The state estimates $\hat{\boldsymbol{s}}_{1, n}$ are used to reestimate the model parameters at time $n$. The recursive model parameter estimator is given below.

Recursive parameter estimation (time $n=1,2, \ldots$ ):

1. $R_{s}^{(n)}(0)=\frac{1}{n-1}\left[(n-2) R_{s}^{(n-1)}(0)+\hat{s}_{1, n} \hat{s}_{1, n}^{\prime}\right]$,

2. $R_{s}^{(n)}(1)=\frac{1}{n-2}\left[(n-3) R_{s}^{(n-1)}(1)+\hat{s}_{1, n-1} \hat{s}_{1, n}^{\prime}\right]$,

3. $\hat{A}_{1}^{(n)}=R_{s}^{(n)}(1) R_{s}^{(n)}(0)^{-1}$,

4. $e_{n}=\hat{s}_{1, n}-\hat{A}_{1}^{(n)} \hat{s}_{1, n-1}$,

5. $\hat{Q}_{1}^{(n)}=\frac{1}{n-1}\left[(n-2) \hat{Q}_{1}^{(n-1)}+\boldsymbol{e}_{n} \boldsymbol{e}_{n}^{\prime}\right]$.

Here, we have assumed that a sufficient amount of training data is available to initialize $R_{s}^{(0)}(0)$ and $R_{s}^{(0)}(1)$. In practice, some information maybe available to initialize or train the mobility estimator. For example, our mobility tracking method maybe used alongside GPS to cover the holes in satellite coverage; in this case, the GPS data may provide training samples for the mobility estimator. We can also use relationships between position, velocity, and acceleration, as in (3), to initialize $\hat{A}_{1}^{(0)}$. Initialization of parameter estimation without any training data is also discussed in Section 5 .

\subsection{Position-AR Parameter Estimation}

As discussed above, the Position-AR mobility model is completely specified by the covariance matrix $Q_{2}$ of the noise. The MMSE estimators for $Q_{2}$, denoted by $\hat{Q}_{2}$, can be obtained from (13) when residual error is defined as $e_{i} \triangleq s_{2, i}-A_{2} s_{2, i-1}$. Similarly, the recursive estimation for noise covariance matrix designed for the AR-1 model can be used with the Position-AR covariance matrix when the residual errors are calculated with $A_{2}$ and $\hat{s}_{2, n}$ and the initial estimate $\hat{Q}_{2}^{(0)}$ is determined from a set of training samples as discussed above.

\section{Mobility Tracking Scheme}

Our proposed mobility tracking scheme consists of the parameter estimation algorithms discussed in Section 3 combined with mobility state estimation, which we shall

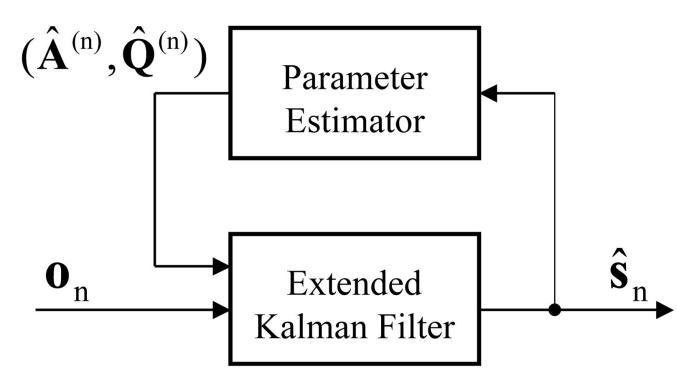

Fig. 1. Mobility tracking via an integrated mobility state and parameter estimator.

discuss in this section. The integrated mobility tracking scheme is shown in Fig. 1. In Fig. 1, the state estimate $\hat{s}_{n}$ could be $\hat{s}_{1, n}$ or $\hat{s}_{2, n}$ depending on whether the underlying mobility model is AR-1 or Position-AR. Similarly, $\hat{Q}$ could be $\hat{Q}_{1}$ or $\hat{Q}_{2}$ and $\hat{A}$ could be $\hat{A}_{1}$ or $A_{2}$, since the transformation matrix is fixed in the case of the PositionAR model. Although not explicitly shown in Fig. 1, the Kalman filter includes a prefilter (cf. [33]) to reduce the effects of measurement noise.

\subsection{Observation Data in Wireless Networks}

To perform mobility state estimation, we assume that either received signal strength indicators (RSSIs) or time of arrival (TOA) measurements from at least three base stations are available. We remark that the angle of arrival (AOA) of the mobile's signal at multiple base stations is often used for location tracking [34], [35], [36]. The AOA is typically estimated using antenna arrays at the base station. However, AOA information is not suitable for use in conjunction with an extended Kalman filter, since the AOA measurements are noncontinuous functions of mobility state that are generally not differentiable. Another measurement used to locate the mobile callers is the time difference of arrival (TDOA) of the signals from two base stations [34], [37], [38]. However, calculation of the TDOA requires time synchronization of the base stations. Moreover, when three base stations are used to provide TDOA measurements, there is often more than one localization solution and, as observed in [34], [39], there is no way to determine the correct solution without the help of additional information, e.g., additional TOA measurements as suggested by [39].

\subsubsection{Pilot Signal Strengths}

In a wireless cellular network, the distance between the mobile and a reachable base station can be inferred from the RSSI or pilot signal strength from the base station. Pilot signal strengths are more readily available in wireless networks than TOA, TDOA, and AOA signals, which require specialized infrastructure to collect useful data. On the other hand, the effectiveness of using RSSI to infer distance information depends on the accuracy of the signal propagation model. The lognormal shadow fading model has proved to be accurate for a large class of urban and suburban environments [40]. According to this model, the RSSI, in units of $\mathrm{dB}$, received at the mobile unit from base station $i$, located at position $\left(a_{i}, b_{i}\right)$ at time $n$ is given as follows [41]:

$$
p_{n, i}=\kappa_{i}-10 \gamma \log \left(d_{n, i}\right)+\psi_{n, i}
$$


where $\kappa_{i}$ is a constant determined by the transmitted power, antenna height, wavelength, and gain of the base station $i, \gamma$ is a slope index (typically, $\gamma$ is between 2 and 5 ), $\psi_{n, i}$ is a zero mean, stationary Gaussian process with standard deviation $\sigma_{\psi}$ typically from 4 to $8 \mathrm{~dB}$, and $d_{n, i}$ is the distance between the mobile node and base station $i$ :

$$
d_{n, i}=\sqrt{\left(x_{n}-a_{i}\right)^{2}+\left(y_{n}-b_{i}\right)^{2}} .
$$

The noise process $\psi_{n, i}$ models shadowing or slow fading, while fast fading is neglected in (14), under the assumption that it is attenuated sufficiently via low-pass filtering (cf. [18], [42]).

Distance measurements to three independent base stations are sufficient to locate the mobile unit in the twodimensional plane. For mobility estimation based on RSSI information, we construct an observation vector consisting of the three largest RSSI measurements, denoted by $p_{n, 1}$, $p_{n, 2}, p_{n, 3}$, as follows:

$$
\boldsymbol{o}_{n}=\left(p_{n, 1}, p_{n, 2}, p_{n, 3}\right)^{\prime}=h\left(\boldsymbol{s}_{n}\right)+\boldsymbol{\psi}_{n},
$$

where $s_{n}$ could be the mobility state defined for either the AR-1 or the Position-AR model, $\psi_{n}=\left(\psi_{n, 1}, \psi_{n, 2}, \psi_{n, 3}\right)^{\prime}$, and

$$
h\left(\boldsymbol{s}_{n}\right)=\kappa-10 \gamma \log \left(\boldsymbol{d}_{n}\right),
$$

where $\kappa=\left(\kappa_{1}, \kappa_{2}, \kappa_{3}\right)^{\prime}$ and $\boldsymbol{d}_{n}=\left(d_{n, 1}, d_{n, 2}, d_{n, 3}\right)^{\prime}$. The covariance matrix of $\psi_{n}$ is given by $R_{\psi}=\sigma_{\psi}^{2} I_{3}$ where $I_{3}$ is the $3 \times 3$ identity matrix.

\subsubsection{Time of Arrival}

Time-based methods of geolocation using TOA and TDOA measurements rely on accurate estimates of the time of arrival of the signals received at several base stations from the mobile station or at the mobile station from several base stations [34], [37]. Several approaches have been developed for estimation of these parameters from received signals, such as code tracking and acquisition in spread spectrum systems using delay-locked loop (DLL) or tau-dither loop as described in [34]. In the presence of measurement noise, the time delay estimate of the signal $\tau_{n, i}$, from base station $i$ measured at the mobile station, at time instant $n$ using DLL is given by

$$
\tau_{n, i}=d_{n, i} / c+\psi_{n, i},
$$

where $d_{n, i}$ is given in (15), $c$ is the speed of light. In contrast to the RSSI model (14), here $\psi_{n, i}$ represents zero mean, white Gaussian noise, with a typical standard deviation of $\sigma_{\psi}=1 \mu \mathrm{s}$ [43].

As in the case of pilot signal strengths, three TOA measurements to neighboring base stations are sufficient for mobility state estimation. The observation vector for TOAbased mobility estimation consists of the three TOA measurements, denoted by $\boldsymbol{o}_{n}=\left(\tau_{n, 1}, \tau_{n, 2}, \tau_{n, 3}\right)^{\prime}$. In this case, the observation equation is given by

$$
\boldsymbol{o}_{n}=h\left(\boldsymbol{s}_{n}\right)+\psi_{n}
$$

where $s_{n}$ could be the mobility state defined for either the AR-1 or the Position-AR model, $\psi_{n}=\left(\psi_{n, 1}, \psi_{n, 2}, \psi_{n, 3}\right)^{\prime}$, and $h\left(s_{n}\right)=d_{n} / c$, where $\boldsymbol{d}_{n}=\left(d_{n, 1}, d_{n, 2}, d_{n, 3}\right)^{\prime}$. As in (16), the covariance matrix of $\psi_{n}$ is denoted by $R_{\psi}=\sigma_{\psi}^{2} I_{3}$.

\subsection{Application of Kalman Filter}

To apply the extended Kalman filter for state estimation, the observation equation given in (16) and (19) can linearized as follows:

$$
\boldsymbol{o}_{n}=h\left(\boldsymbol{s}_{n}^{*}\right)+H_{n} \Delta \boldsymbol{s}_{n}+\boldsymbol{\psi}_{n},
$$

where $s_{n}^{*}$ is the nominal or reference vector and $\Delta s_{n}=$ $s_{n}-s_{n}^{*}$ is the difference between the true and nominal state vectors. In the extended Kalman filter (cf. [24]), the nominal vector is obtained from the estimated state trajectory, $\hat{s}_{n}$, i.e., $s_{n}^{*}=\hat{s}_{n}$. The matrix $H_{n}$ is given by

$$
H_{n}=\left.\frac{\partial h}{\partial \boldsymbol{s}}\right|_{s=\hat{s}_{n}} .
$$

Expressions for the matrix $H_{n}$, for both RSSI and TOA measurements, are given in the Appendix.

Let $o_{i}^{j}=\left(\boldsymbol{o}_{i}, \ldots, \boldsymbol{o}_{j}\right)$ denote the set of observations from time $i$ to time $j$ for $j>i$. The Kalman filter estimates are denoted as follows:

$$
\hat{\boldsymbol{s}}_{n \mid n}=E\left[\boldsymbol{s}_{n} \mid \boldsymbol{o}_{1}^{n}\right] \text { and } \hat{\boldsymbol{s}}_{n \mid n-1}=E\left[\boldsymbol{s}_{n} \mid \boldsymbol{o}_{1}^{n-1}\right] .
$$

The covariance matrices corresponding to these estimates are denoted by

$$
M_{n \mid n}=\operatorname{Cov}\left[s_{n} \mid \boldsymbol{o}_{1}^{n}\right] \text { and } M_{n+1 \mid n}=\operatorname{Cov}\left[\boldsymbol{s}_{n+1} \mid \boldsymbol{o}_{1}^{n}\right],
$$

respectively. The Kalman filter procedure for state estimation is then given as follows:

Mobility state estimation (time $n=2,3, \ldots$ ):

1. $H_{n}=\left.\frac{\partial h}{\partial s}\right|_{s=\hat{s}_{n}}$

2. $K_{n}=M_{n \mid n-1} H_{n}^{\prime}\left(H_{n} M_{n \mid n-1} H_{n}^{\prime}+R_{\psi}\right)^{-1}$,

3. $\hat{\boldsymbol{s}}_{n \mid n}=\hat{\boldsymbol{s}}_{n \mid n-1}+K_{n}\left(\boldsymbol{o}_{n}-h\left(\hat{\boldsymbol{s}}_{n \mid n-1}\right)\right)$ [Correction step],

4. $M_{n \mid n}=\left(I-K_{n} H_{n}\right) M_{n \mid n-1}\left(I-K_{n} H_{n}\right)^{\prime}-K_{n} R_{\psi} K_{n}^{\prime}$

5. $\hat{\boldsymbol{s}}_{n+1 \mid n}=\hat{A}^{(n)} \hat{\boldsymbol{s}}_{n \mid n}$ [Prediction step],

6. $M_{n+1 \mid n}=\hat{A}^{(n)} M_{n \mid n} \hat{A}^{(n)^{\prime}}+\hat{Q}^{(n)}$.

In the above procedure, the matrices $\hat{A}^{(n)}$ and $\hat{Q}^{(n)}$ correspond to the $n$th estimate of the transformation and covariance matrices of both mobility models, as indicated in Fig. 1. For the Position-AR model, the matrix $\hat{A}^{(n)}$ is replaced by the constant matrix in (7). The matrix $K_{n}$ is referred to as the Kalman gain matrix. The mobility state estimate at time $n$ is then defined by $\hat{s}_{n} \triangleq \hat{s}_{n \mid n}$. The initialization of the Kalman filter is specified by

$$
\hat{s}_{1 \mid 0}=E\left[s_{1}\right] \text { and } M_{1 \mid 0}=\operatorname{Cov}\left[s_{1}\right] .
$$

In practice, we set the initial parameters for both mobility models as follows:

$$
\hat{\boldsymbol{s}}_{1 \mid 0}=\left(\hat{x}_{1}, 0,0, \hat{y}_{1}, 0,0\right)^{\prime} \text { and } M_{1 \mid 0}=I_{6},
$$

where $\left(\hat{x}_{1}, \hat{y}_{1}\right)$ is the best available estimate of the initial position of the mobile unit. In practice, some information maybe available to initialize the mobility estimator. For example, knowledge of the approximate location of the mobile user, e.g., the median coordinate values of the cell sector in which the user resides, could be used to initialize the mobility state vector. As another example, our mobility tracking method maybe used alongside GPS to cover the 
holes in satellite coverage; in this case, the GPS data may provide initial state vector along with training data for model parameter estimation.

\subsection{Relationship to EM Algorithm and Convergence}

The mobility tracking scheme of Fig. 1 is related to the Expectation-Maximization (EM) algorithm (cf. [44]). The E-step of the EM algorithm corresponds to the estimation of a hidden state sequence from observations (or incomplete data) by finding (cf. [44])

$$
\hat{\boldsymbol{s}}_{n}=E\left[\boldsymbol{s}_{n} \mid \boldsymbol{o}_{1}^{n}, \theta_{n-1}\right],
$$

where $\theta_{n}$ denotes the $n$th estimate of the unknown model parameters, e.g., $\hat{A}^{(n)}$ and $\hat{Q}^{(n)}$ for the AR-1 model and $\hat{Q}^{(n)}$ for the Position-AR mobility model. Equation (22) holds for the Kalman filter [24], and holds approximately for the extended Kalman filter, provided the difference between the true and nominal trajectory, i.e., $\Delta s_{n}=s_{n}-s_{n}^{*}=s_{n}-\hat{s}_{n \mid n-1}$, remains small.

The M-step of the EM algorithm [44] relates to parameter estimation. Let $\hat{\theta}_{n}$ denote the $n$th estimate of an unknown parameter $\theta$. In the M-step, $\hat{\theta}_{n}$ is computed such that it maximizes the expectation of the log-likelihood of the mobility state and observation sequences, given the observations and the current parameter estimate [44], i.e.,

$$
\hat{\theta}_{n}=\arg \max _{\theta} E\left[\log p\left(s_{1}^{n}, \boldsymbol{o}_{1}^{n} \mid \theta\right) \mid \boldsymbol{o}_{1}^{n}, \hat{\theta}_{n-1}\right],
$$

where $s_{1}^{n}=\left(s_{1}, \ldots, s_{n}\right)$ denotes the sequence of mobility states from time 1 to $n$. Using the first-order autoregressive property of the AR-1 and Position-AR mobility models and the assumption that (22) holds, the M-step in (23) can be written as

$$
\begin{aligned}
\hat{\theta}_{n}= & \arg \max _{\theta}\left[-\frac{n}{2} \log \left((2 \pi)^{6}|Q|\right)\right. \\
& \left.-\frac{1}{2} \sum_{i=1}^{n}\left(\hat{\boldsymbol{s}}_{i}-A \hat{\boldsymbol{s}}_{i-1}\right) Q^{-1}\left(\hat{\boldsymbol{s}}_{i}-A \hat{\boldsymbol{s}}_{i-1}\right)^{\prime}\right] .
\end{aligned}
$$

The maximization in (24) yields an estimate for $A$ of the form (10) and an estimate for $Q$ of the form (13).

As long as $\Delta s_{n}$ is kept small, convergence properties for the EM algorithm carry over to the proposed mobility tracking scheme. In particular, it can be shown that under certain conditions, the estimates for $A$ and $Q$ will converge to a stationary point of the likelihood function, which could be a global or local maximum or a saddle point (cf. [45, Section 2.1]). Convergence of the mobility state estimator depends on issues related to extended Kalman filters. An important factor to reduce numerical roundoff errors is to initialize the filter with proper initial estimates (cf. [24, pp. 260-264, 346]). Convergence of Kalman filters is also dependent on the observability of the system [24]. In [33], the observability issue was investigated in the case of the linear dynamic system model. Similar analysis for the AR- 1 and Position-AR models shows that the extended Kalman filter is observable under fairly general conditions when three or more observations are used.

\section{Numerical Results}

In this section, we present some representative numerical results to validate the effectiveness of the AR- 1 and PositionAR models and the associated mobility tracking schemes. We apply both models to mobility patterns obtained from drive tests, as well as those generated by alternative mobility models such as the random waypoint and linear system models and compare the respective estimation results under various operating conditions.

\subsection{Data Collection}

We collected three sets of drive test GPS location data containing more than 1,200 sample points each. One set of data was collected from a suburban area while another set was obtained from a downtown city environment with an orthogonal street layout. The third drive test was carried out by a walking subject in the Fairfax campus of George Mason University (GMU). The drive test data consisted of a sequence of $(x, y)$-coordinates characterizing the trajectory of the mobile user. For each data set, we construct a corresponding mobility state sequence $\left\{s_{1}, \ldots s_{N_{T}}\right\}$ for both models, where $N_{T}$ denotes the total number of data points.

The data of interest, collected from the drive test, consisted of latitude and longitude values of the mobile user at predefined measurement time intervals. The drive test was converted from latitude/longitude (Lat/Long) coordinates, in decimal format, to two-dimensional Cartesian coordinates $(x, y)$ as follows:

$$
\begin{aligned}
x & =C \frac{\pi \cos \left(\mathrm{Lat}_{0}\right)}{180}\left(\mathrm{Long}_{0}-\mathrm{Long}\right), \\
y & =C \frac{\pi}{180}\left(\text { Lat }-\mathrm{Lat}_{0}\right),
\end{aligned}
$$

where $C=6,378,137$ and $\left(\mathrm{Lat}_{0}, \mathrm{Long}_{0}\right.$ ) correspond to the latitude and longitude, in decimal format, of the origin $(0,0)$ of the local Cartesian coordinate system. Using the GPS data, RSSI and TOA measurement data were generated using (14) and (18), respectively, with fixed parameter values for all drive test scenarios. The simulated observation data along with the drive test trajectories allowed us to conduct a detailed performance study of the proposed mobility estimators and their dependence on various factors and operational settings.

For the urban scenario only, we were able to collect three or more RSSI measurements for every position sample, which allowed us to perform mobility tracking based on real RSSI data rather than simulated RSSI data generated from GPS location data. This result is shown in Fig. 4, which demonstrates the effectiveness of the proposed mobility tracking scheme in the presence of propagation modeling error in a challenging urban environment. As discussed above, the other mobility tracking experiments based on the drive test data were performed using simulated RSSI and TOA measurement data generated from the GPS data.

\subsection{Validation of Mobility Model}

To validate the accuracy of the mobility model, we use the multiple coefficient of determination, denoted by $R^{2}$ (cf. 
TABLE 1

$R^{2}$ Values for Sample Data Sets

\begin{tabular}{c|c|cc}
\hline \hline & Training & \multicolumn{2}{c}{$R^{2}$} \\
Damples & AR-1 & Position-AR \\
\hline suburban (driving) & 100 & 0.99 & 1.0 \\
urban (driving) & 500 & 0.95 & 1.0 \\
GMU (walking) & 100 & 0.94 & 0.99 \\
\hline \hline
\end{tabular}

[46], [47]). For each of the mobility state sequences, we use the first $N$ mobility states as training samples to obtain estimates $\hat{A}_{1}$ and $\hat{Q}_{1}$ for the AR- 1 model and $\hat{Q}_{2}$ for the Position-AR model, as discussed in Section 3. The remaining mobility states, $s_{N+1}, \ldots, s_{N_{T}}$, are then used to compute the $R^{2}$ metric as follows:

$$
R^{2}=1-\frac{\sum_{i=N+1}^{N_{T}}\left|s_{i}-A s_{i-1}\right|^{2}}{\sum_{i=N+1}^{N_{T}}\left|s_{i}-\bar{s}\right|^{2}}
$$

where $s_{i}$ is the $i$ th mobility state for either model and

$$
\bar{s}=\frac{1}{N_{T}-N} \sum_{i=N+1}^{N_{T}} s_{i}
$$

is the sample average of the mobility state sequence after the first $N$ states. The value of $R^{2}$ always lies in the interval $[0,1]$. A value of $R^{2}$ close to 1 indicates a strong model fit. Table 1 shows the $R^{2}$ values for the three data sets for the AR-1 and Position-AR models. The results demonstrate that statistically accurate mobility characterizations in terms of the AR-1 and Position-AR models can be achieved.

The sampling interval of one second is relatively short compared to the dynamics of our drive test trajectories. Hence, each of the sampled trajectories closely follows the corresponding actual trajectory even though the mobility trace may appear to be highly nonlinear. Consequently, both the AR-1 and Position-AR mobility estimators perform well with respect to the $R^{2}$ metric, as shown in Table 1 . Note that slightly lower $R^{2}$ value is obtained for the walking scenario compared to the suburban scenario. Similarly, compared to the suburban and walking scenarios, five times the number of training samples is needed in the urban scenario to achieve an $R^{2}$ value of 0.95 or higher for both estimators. These results can be explained in terms of Fig. 5, which visually shows that both the walking and urban trajectories contain more turns and bends than the suburban trajectory.

The noise terms in the AR-1 and Position-AR models are assumed to be zero mean white Gaussian noise processes. To check the validity of this assumption, we use residual error analysis (cf. [47]). The residual error for a data point $s_{i}$ is defined as $e_{i}=s_{i}-\hat{s}_{i}$. The plots in Fig. 2 show that the residual errors in the $x$ and $y$ dimensions seem to be independent of the data points. These plots were generated for the Position-AR model using the suburban trajectory data with model parameter estimation as described in Section 3. Other data sets also show similar characteristics. Fig. 3 shows two Q-Q plots (cf. [47]) for residual errors for
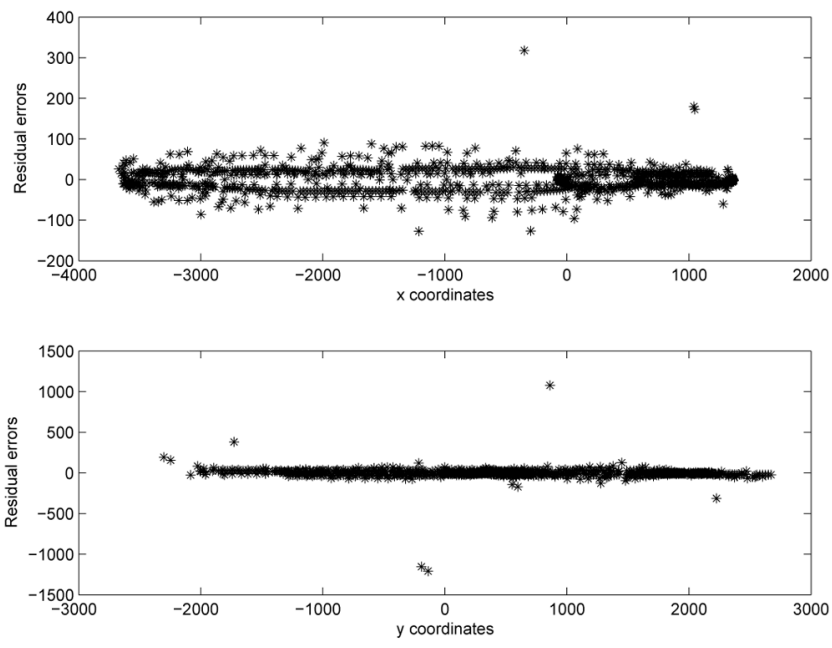

Fig. 2. Independence of residual errors, Position-AR model.

the Position-AR model in the $x$ and $y$ dimensions. The close straight-line fit observed in both plots confirms the validity of the assumption that the residual errors or model noise can be modeled accurately by white Gaussian processes. Similar graphs for the AR-1 model are presented in [23].

\subsection{Validation of Mobility Estimation Scheme}

As discussed in Section 5.1, we were able to obtain a sufficient quantity of RSSI measurements in one set of drive test data collected from the urban scenario. Although one experiment is not sufficient to provide a comprehensive validation of our mobility estimation scheme, it shows the feasibility of our method in a real-world setting. Since the parameters of (14) are not known for the RSSI observation data collected in the urban scenario, we employ least-squares estimation to determine $\kappa_{i}, \gamma$, and $\sigma_{\psi}$ for all base stations. In the urban scenario drive test experiment, 41 base stations supplied 3 or more RSSI values after every 2 second interval. The least-squares estimates $\hat{\kappa}_{i}, \hat{\gamma}$, and $\hat{\sigma_{\psi}}$ for propagation model parameters are given as follows:
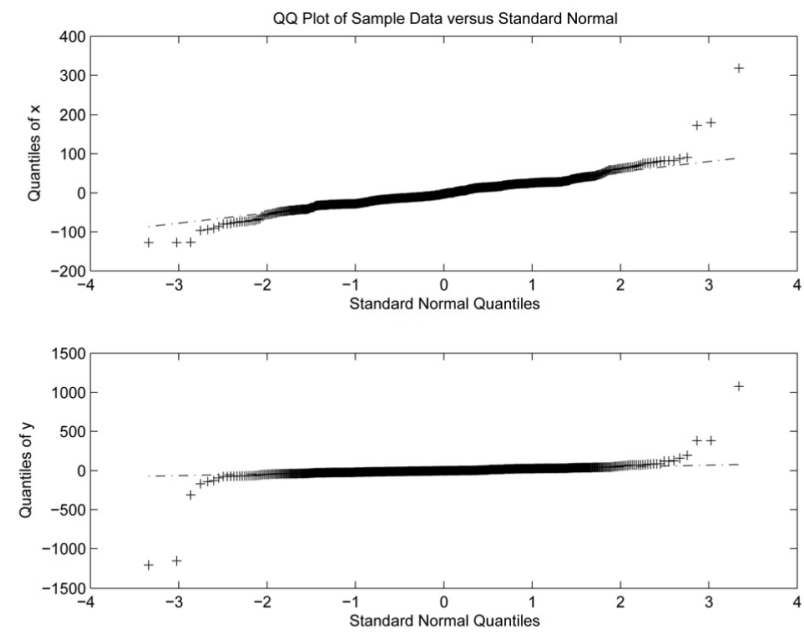

Fig. 3. Q-Q plots for residual error, Position-AR model. 


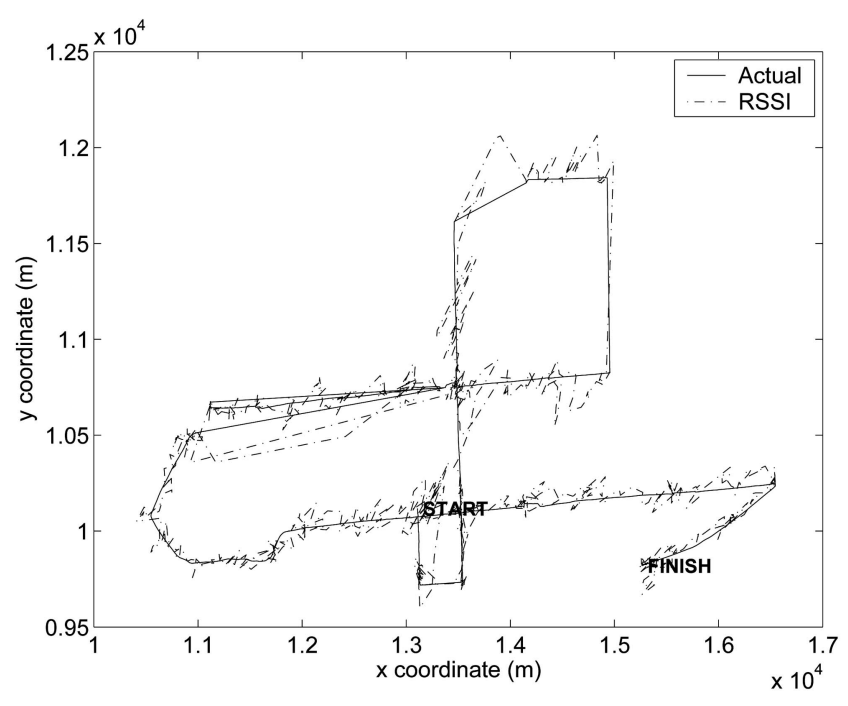

Fig. 4. Actual and estimated trajectories using AR-1 model and real RSSI data obtained from urban scenario.

$$
\begin{aligned}
\hat{\kappa_{i}} & =\frac{1}{N} \sum_{n=1}^{N}\left(p_{n, i}+10 \hat{\gamma} \log d_{n, i}\right), \\
\hat{\gamma} & =\frac{1}{N} \frac{\sum_{n=1}^{N} p_{n, i} \log d_{n, i}-\frac{1}{N} \sum_{n=1}^{N} p_{n, i} \sum_{n=1}^{N} \log d_{n, i}}{\sum_{n=1}^{N}\left(\log d_{n, i}\right)^{2}-\frac{1}{N}\left(\sum_{n=1}^{N} \log d_{n, i}\right)^{2}} \\
\hat{\sigma_{\psi}} & =\frac{1}{N} \sum_{n=1}^{N}\left(p_{n, i}-\hat{\kappa}_{i}+10 \hat{\gamma} \log d_{n, i}\right)^{2} .
\end{aligned}
$$

Here, the value of $N$ is selected to be 10 to allow the parameter estimates adapt to the dynamic changes in the urban propagation environment. The performance of mobility tracking based on the AR-1 model in this scenario is shown in Fig. 4, where the initial 300 samples of the drive test trajectory are used as training samples.

Next, we generated simulated observation data from the drive test trajectories in all scenarios to validate the effectiveness of the mobility estimation scheme based on the AR-1 and Position-AR models (cf. Section 4). The use of simulated observation data allowed us to study the effects of different operational settings, such as number of training samples and cell size, on mobility tracking performance. We assume that the service area is subdivided into a rectangular grid with square cells. Each cell contains one base station located in the center of the cell. In our simulation experiments, a mobile user moving along a drive test trajectory receives signals (either RSSI or TOA) from the base stations and employs the integrated mobility estimator to determine the mobility state as well as the parameters of the underlying AR-1 or Position-AR model. A training set consisting of the first few data points in the actual mobility state sequence is used to obtain the initial estimates $\hat{A}_{1}$ and $\hat{Q}_{1}$ of the AR- 1 model and $\hat{Q}_{2}$ of the Position-AR model.

The RSSI measurements are generated using (14) with the parameter $\kappa$ assumed to be zero for all base stations and $\gamma$ set to 5 for all scenarios. Typical values for the shadowing noise standard deviation, i.e., $\sigma_{\psi}$, range from 4 to $8 \mathrm{~dB}$ [41]. The shadowing standard deviation is taken as $8 \mathrm{~dB}$. The TOA measurements are generating using (18). The error noise in the TOA measurements is assumed to be a white Gaussian process with a standard deviation of $\sigma_{\psi} \approx 1 \mu \mathrm{s}$ [43]. To reduce measurement noise, two prefilters are applied to the observation data prior to the extended Kalman filter (cf. [33]).

Fig. 5 depicts the mobile trajectories obtained from three drive test scenarios: urban, suburban, and walking. The cell size is approximated by a $1 \mathrm{~km} \times 1 \mathrm{~km}$ square and 100 initial samples are used as training data in all scenarios. The mobility state estimation procedure generates a sequence of mobility state estimates $\left\{\hat{s}_{2}, \ldots, \hat{s}_{N}\right\}$. Fig. 5 also shows, for each scenario, the estimated trajectory obtained using the AR-1 mobility estimator with TOA observations.

The sequence of position estimates $\left\{\left(\hat{x}_{n}, \hat{y}_{n}\right)\right\}$ can be compared quantitatively against the sequence of actual positions $\left\{\left(x_{n}, y_{n}\right)\right\}$ in terms of root-mean-squared error (RMSE) as a figure of merit, defined by

$$
\mathrm{RMSE}=\sqrt{\frac{1}{N-1} \sum_{n=2}^{N}\left[\left(\hat{x}_{n}-x_{n}\right)^{2}+\left(\hat{y}_{n}-y_{n}\right)^{2}\right] .}
$$

Fig. 6 shows the RMSE performance of mobility estimation schemes based on the AR-1 and Position-AR models for three data sets using RSSI and TOA measurements when different number of training samples are used to initialize

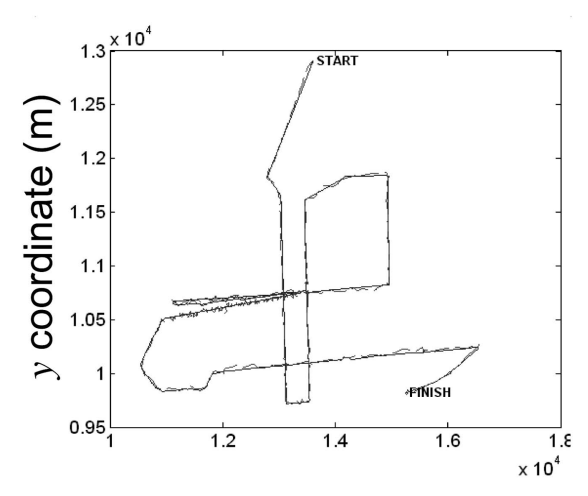

(a)

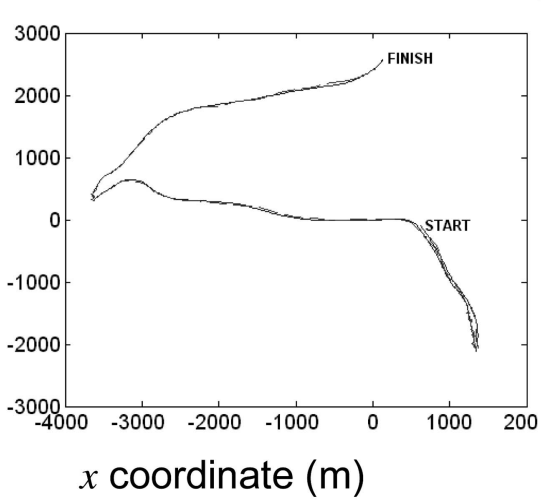

(b)

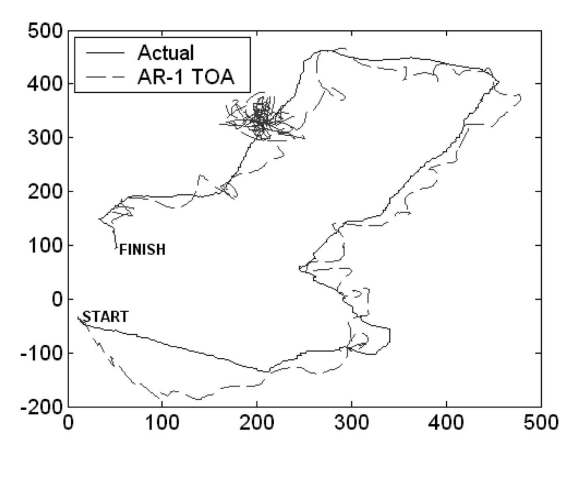

(c)

Fig. 5. Actual and estimated trajectories using AR-1 model and TOA observations in (a) urban, (b) suburban, and (c) GMU walking scenarios. 


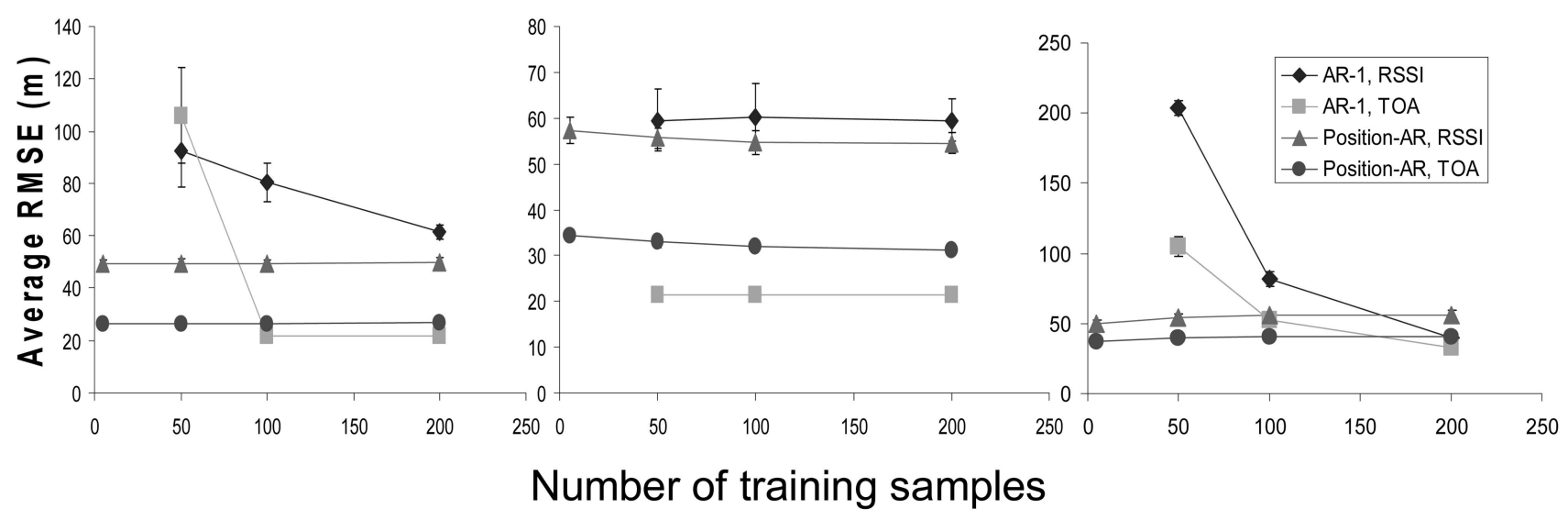

(a)

(b)

(c)

Fig. 6. RMSE performance versus number of training samples for (a) urban, (b) suburban, and (c) GMU walking scenarios.

model parameters. Each point in the graphs of Fig. 6 shows the average RMSE, i.e., $\mu_{\mathrm{RMSE}}$, over 50 experiments and the error bars represent $\pm \sigma_{\mathrm{RMSE}}$ for each point. Line segments connecting the data points are only used to enhance the clarity of the plots. The cell size is approximated by a $1 \mathrm{~km} \times 1 \mathrm{~km}$ square.

From the graphs of Fig. 6, we observe that more training samples result in better RMSE performance for the scenarios of our study. A training set which is an accurate representation of the trajectory would be an ideal choice for parameter initialization. However, we are choosing the first few samples of the trajectories which might not represent the whole trajectories accurately. This difference is evident in Fig. 6, where the mobility tracking results for the suburban trajectory are smoother than those of the rest of the data sets as it is mostly comprised of straight-line segments with fewer bends. The urban data set was obtained from a downtown area with orthogonal streets and walking scenarios is also comprised of more curved segments. In such scenarios, a larger training set is recommended.

We also note that the Position-AR model requires the estimation of fewer parameters than the AR-1 model and hence requires fewer training samples for proper initialization. Furthermore, the Position-AR model shows stable performance when the training set size is varied, in contrast to the AR-1 model, which performs poorly for small training sets. With a sufficient training set, the RMSE performance of the AR-1 model can be better than that of the Position-AR model, as the matrix $A_{1}$ in the AR- 1 model adapts to be changed in the mobility characteristics. With typical values of noise variance, the mobility tracking performance with TOA measurements was superior to that with RSSI measurements for all scenarios considered in this study.

Table 2 shows the performance of mobility tracking based on the AR-1 and Position-AR models when no training data are provided. In both models, the noise covariance matrices $\hat{Q}_{1}^{(0)}$ and $\hat{Q}_{2}^{(0)}$ are each initialized to the $6 \times 6$ identity matrix and the initial state vector estimate, $\hat{s}_{1 \mid 0}$, is initialized to the zero vector. For the AR-1 model, the transformation matrix is initialized as follows, using similar relationships as in (3):

$$
\hat{A}_{1}^{(0)}=\left[\begin{array}{cc}
A_{x} & 0_{3 \times 3} \\
0_{3 \times 3} & A_{y}
\end{array}\right],
$$

where

$$
A_{x}=A_{y}=\left[\begin{array}{ccc}
1 & T & T^{2} / 2 \\
0 & 1 & T \\
0 & 0 & \alpha
\end{array}\right],
$$

$T$ is the sampling interval, and $\alpha$ is given an arbitrary value of 0.5 . We have removed the first 50 estimated location points from RMSE calculations to focus on the steady state behavior of the mobility estimators. From Table 2, one sees that even without training data, the mobility estimator based on the Position-AR model tracks the mobile user with reasonable accuracy. By contrast, the AR-1 based mobility estimator produces large errors, especially when RSSI observations are used.

The last factor considered in our study is cell size, to show the effect of observation data from distant base stations. Training data size is kept at 100 samples for all data sets and two filters are used in the prefiltering step (cf. [33]) for this experiment. Fig. 7 shows the RMSE performance for different cell sizes. The x-axis in Fig. 7 shows the length of each side of the square cell. An increase in cell size generally reduces estimation accuracy. TOA-based mobility tracking outperforms RSSI-based tracking in all of our experiments. Also, Fig. 7 shows that tracking based on TOA measurements is more resilient than that based on RSSI when distances between the mobile user and the base stations increase.

TABLE 2

RMSE of Estimation Algorithms When No Training Data are Available

\begin{tabular}{c|cc|cc}
\hline \hline & \multicolumn{2}{|c|}{ RSSI } & \multicolumn{2}{c}{ TOA } \\
\hline Scenarios & AR-1 & Position-AR & AR-1 & Position-AR \\
& $\left(\mu_{\text {RMSE }}, \sigma_{\text {RMSE }}\right)$ & $\left(\mu_{\text {RMSE }}, \sigma_{\text {RMSE }}\right)$ & $\left(\mu_{\text {RMSE }}, \sigma_{\text {RMSE }}\right)$ & $\left(\mu_{\text {RMSE }}, \sigma_{\text {RMSE }}\right)$ \\
& $(\mathrm{m})$ & $(\mathrm{m})$ & $(\mathrm{m})$ & $(\mathrm{m})$ \\
\hline Urban & $(5243.1,143.1)$ & $(48.28,2.45)$ & $(612.6,1310.8)$ & $(23.08,0.54)$ \\
Suburban & $(2246.4,5.4)$ & $(55.16,2.7)$ & $(22.5,3.14)$ & $(32.97,1.02)$ \\
GMU walking & $(373.48,0)$ & $(51.26,2.3)$ & $(373.04,3.1)$ & $(38.43,1.2)$ \\
\hline \hline
\end{tabular}




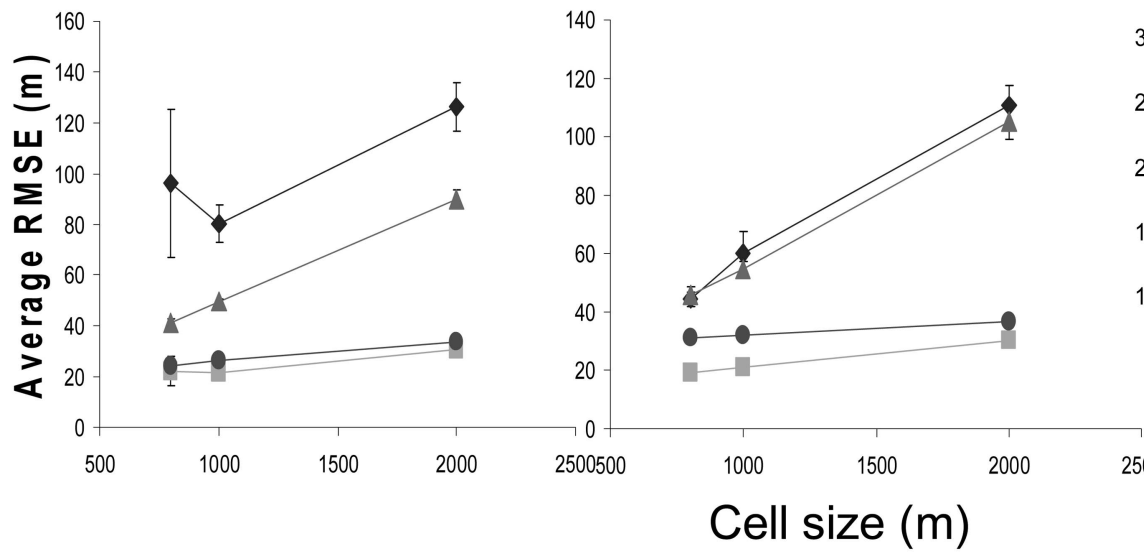

(a) (b)

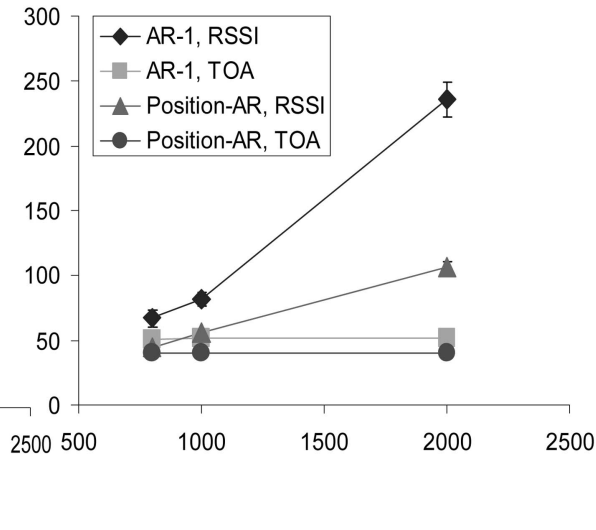

(c)

Fig. 7. RMSE performance versus cell size for (a) urban, (b) suburban, and (c) GMU walking scenarios.

The simulation results show that the mobility state estimators performed with reasonable accuracy under the three different mobility scenarios with appropriate selection of operating conditions. In general, a larger training set is preferable if available and the three largest signal measurements should be chosen among all available values. A training phase could also be used to bootstrap the operational settings of the mobility estimation scheme, e.g., the number of prefilters (cf. [33]), noise covariance value, etc.

\subsection{Comparison with Other Mobility Models}

The AR-1 and Position-AR models can accurately represent trajectories generated by other stochastic mobility models. Models such as the random waypoint model may be used for generating mobility patterns in simulation environments, but generally cannot be used to track mobility in real time as it is not straightforward to estimate the model parameters for real scenarios. The linear system model of mobility, discussed in [18], can be used to develop a mobility state estimator [33]. However, the parameters of the model cannot be estimated in an optimal way. The estimator performs well only if the model parameters represent accurately the dynamic behavior of the mobile unit.

We generated trajectories, containing more than 1,000 data points, using the linear dynamic system model [18], [19] and the random waypoint model [4], [48]. We then used the proposed mobility estimation scheme to track the generated trajectories. In applying the mobility estimator, we assumed that training sets of 100 data points for the linear system model trajectory and 300 samples for the random waypoint trajectory were available to initialize the AR- 1 and PositionAR models. The cell size was set as $1,000 \mathrm{~m} \times 1,000 \mathrm{~m}$ and two prefilters are used prior to the extended Kalman filter (cf. [33]).

The parameters of the linear system model of mobility were set as follows (cf. [18], [19]): $\alpha=1,000 \mathrm{~s}^{-1}, T=1 \mathrm{~s}$, and $\sigma_{1}=1 \mathrm{~dB}$. The discrete command processes $u_{x}(t)$ and $u_{y}(t)$ are independent semi-Markov processes, each of which was assumed to take on five possible levels of acceleration comprising the set $\{-0.5,-0.25,0,0.25,0.5\}$ in units of $\mathrm{m} / \mathrm{s}^{2}$. The initial probability vector $\pi$ for the semi-Markov model
(SMM) governing $u_{x}(t)$ and $u_{y}(t)$ was initialized to a uniform distribution. The elements of the transition probability matrix for the SMM were initialized to a common value of $1 / 5$. We assumed that the dwell times in each state were uniformly distributed with a common mean value of $2 T$. The RSSI and TOA measurements were generated in a similar manner to that specified in the test scenarios.

The sample mean and standard deviation of RMSE statistics collected from 50 simulations are given in Table 3 . The result shows that the estimators based on the AR-1 and Position-AR models were able to obtain appropriate model parameters and accurately track the trajectory generated by the linear dynamic system model with both RSSI and TOA measurements.

We also generated trajectories using the random waypoint model and applied our proposed integrated mobility estimator. The trajectories were generated within an area of $2,000 \mathrm{~m} \times 2,000 \mathrm{~m}$, centered at the origin. The speed of the mobile unit was uniformly distributed in the interval $[0,60] \mathrm{m} / \mathrm{s}^{2}$ and the mean pause time was assumed to be 1 s. The random waypoint model tends to generate trajectories with sharp turns, which are quite different from realistic mobility patterns. Nevertheless, the mobility estimator was able to accurately track the trajectory, as shown in Table 3.

The standard deviation of the RMSE is higher for the linear system model, as shown in Table 3, than for the random waypoint model because the mobility patterns generated by the random waypoint model largely consist of straight-line segments in which the mobile user moves at a constant speed (i.e., zero acceleration). The autoregressivemodel-based estimators are able to track the speed quite

TABLE 3

RMSE of Estimation Algorithm-Stochastic Models

\begin{tabular}{c|cc|cc}
\hline \hline & \multicolumn{2}{|c|}{ RSSI } & \multicolumn{2}{c}{ TOA } \\
\hline Models & AR-1 & Position-AR & AR-1 & Position-AR \\
& $\left(\mu_{\text {RMSE }}, \sigma_{\text {RMSE }}\right)$ & $\left(\mu_{\text {RMSE }}, \sigma_{\text {RMSE }}\right)$ & $\left(\mu_{\text {RMSE, }} \sigma_{\text {RMSE }}\right)$ & $\left(\mu_{\text {RMSE, }} \sigma_{\text {RMSE }}\right)$ \\
& $(\mathrm{m})$ & $(\mathrm{m})$ & $(\mathrm{m})$ & $(\mathrm{m})$ \\
\hline Linear system & $(68.93,8.57)$ & $(52.46,6.8)$ & $(43.29,4.33)$ & $(38.14,3.56)$ \\
Random waypoint & $(49.69,3)$ & $(52.97,4.8)$ & $(21.44,1.2)$ & $(26.28,1.4)$ \\
\hline \hline
\end{tabular}


accurately along these straight-line path segments. Most of the error in the tracking occurs at the waypoints at which a random destination point and speed are chosen according to the random waypoint model. We also performed some experiments with the random waypoint mobility model using different speed distributions, such as a constant speed of $30 \mathrm{~m} / \mathrm{s}$ and exponential distribution with a mean speed of $30 \mathrm{~m} / \mathrm{s}$. In both cases, we observed similar locationtracking performance as shown in Table 3. The estimation performance does not seemed to be affected by the speed distributions chosen for the random waypoint model.

\section{Conclusion}

We proposed a mobility tracking scheme based on two autoregressive models of mobility, which we refer to as the AR-1 and Position-AR models. The AR-1 and Position-AR models are relatively simple, yet to provide more accurate representation of realistic mobility patterns than other mobility models. Both models were validated using mobile trajectory data obtained from a cellular network, as well as simulated data obtained from the random waypoint and linear system models of mobility. The proposed mobility tracking scheme consists of an integrated scheme of MMSE parameter estimation and mobility state estimation based on Kalman filtering using observations of RSSI and TOA from the network. The scheme provides a viable solution to the two important issues of realistic mobility modeling and real-time mobility tracking for wireless networks.

We have also analyzed the effects of different operational settings on the performance of mobility estimation scheme using both models and both observations. The Position-AR model requires a smaller number of training samples for initialization than the AR-1 model and is more resilient to perturbations in operating conditions. With typical values of noise variance for both types of observation data, mobility tracking performance using TOA measurements was superior to the performance using RSSI measurements in our experiments.

The proposed mobility tracking schemes can enable mobility-aware applications, which can improve performance or provide new services in wireless networks. In cellular networks, for example, the mobility estimation scheme could be used to predict cell crossings for smoother handoffs and more efficient resource allocation [18]. The mobility tracking scheme could also be adapted for mobile ad hoc networks (cf. [49]).

\section{APPENDIX}

The matrix $H_{n}$ in (21) is given by $H_{n}=\left(\boldsymbol{h}_{n, 1}^{\prime}, \ldots \boldsymbol{h}_{n, m}^{\prime}\right)^{\prime}$, where $\boldsymbol{h}_{n, i}$ is the $i$ th row of $H_{n}$ for $i=1,2,3$. For RSSI,

$$
h_{n, i}=\frac{-10 \gamma}{\left(d_{n, i}\right)^{2}}\left(x_{n}-a_{i}, 0,0, y_{n}-b_{i}, 0,0\right) .
$$

For TOA,

$$
\boldsymbol{h}_{n, i}=\frac{1}{c\left(d_{n, i}\right)}\left(x_{n}-a_{i}, 0,0, y_{n}-b_{i}, 0,0\right) .
$$

\section{ACKNOWLEDGMENTS}

The authors thank Mohammed Benchaaboune for the drive test data and Dr. Asad Zaman of Lahore University of Management Sciences for his useful feedback. This work was supported in part by the US National Science Foundation under grants ACI-0133390 and ECS-123232.

\section{References}

[1] C. Bettstetter, "Smooth is Better than Sharp: A Random Mobility Model for Simulation of Wireless Networks," Proc. ACM Int'l Conf. Modeling, Analysis and Simulation of Wireless and Mobile Systems (MSWiM), pp. 19-27, July 2001.

[2] T. Camp, J. Boleng, and V. Davies, "Survey of Mobility Models for Ad Hoc Network Research," Wireless Comm. and Mobile Computing, vol. 2, no. 5, pp. 483-502, 2002.

[3] I.F. Akyildiz, Y.B. Lin, W.R. Lai, and R.J. Chen, "A New Random Walk Model for PCS Networks," IEEE J. Selected Areas in Comm., vol. 18, no. 7, pp. 1254-1260, July 2000.

[4] D. Johnson and D. Maltz, "Dynamic Source Routing in Ad Hoc Wireless Networks," Mobile Computing, vol. 353, pp. 153-181, 1996.

[5] H. Stark and J.W. Woods, Probability and Random Processes with Applications to Signal Processing, third ed. Prentice Hall, 2001.

[6] B. Liang and Z.J. Haas, "Predictive Distance-Based Mobility Management for Multidimensional PCS Networks," IEEE/ACM Trans. Networking, vol. 11, no. 5, pp. 718-732, Oct. 2003.

[7] H. Kobayashi, S.Z. Yu, and B.L. Mark, "An Integrated Mobility and Traffic Model for Resource Allocation in Wireless Networks," Proc. Third ACM Int'l Workshop Wireless Mobile Multimedia (WoWMoM), pp. 39-47, Aug. 2000.

[8] J. Yoon, M. Liu, and B. Noble, "Random Waypoint Considered Harmful," Proc. IEEE INFOCOM, pp. 1312-1321, Mar. 2003.

[9] J. Yoon, M. Liu, and B. Noble, "Sound Mobility Models," Proc. ACM MobiCom, pp. 205-216, Sept. 2003.

[10] A. Jardosh, E.M. Belding-Royer, K. Almeroth, and S. Suri, "Towards Realistic Mobility Models for Mobile Ad Hoc Networks," Proc. ACM MobiCom, pp. 217-229, Sept. 2003.

[11] J. Härri, F. Filali, and C. Bonnet, "A Framework for Mobility Models Generation and Its Application to Inter-Vehicular Networks," Proc. IEEE Int'l Conf. Wireless Networks, Comm. and Mobile Computing, vol. 1, pp. 42-47, June 2005.

[12] P.A. Dintchev, B. Perez-Quiles, and E. Bonek, "An Improved Mobility Model for 2G and 3G Cellular Systems," Proc. IEEE 3G Mobile Comm. Technologies, vol. 1, pp. 402-406, 2004.

[13] A. Momen, A. Mirzaee, and S. Masajedian, "An Analytical Random Direction-Based Method in User Mobility Modeling for Wireless Networks," Proc. IEEE 16th Int'l Symp. Personal, Indoor and Mobile Radio Comm. (PIMRC), pp. 2050-2057, Sept. 2005.

[14] G. Lu and G.M.D. Belis, "Study on Environment Mobility Models for Mobile Ad Hoc Network: Hotspot Mobility Model and Route Mobility Model," Proc. IEEE Int'l Conf. Wireless Networks, Comm. and Mobile Computing, vol. 1, pp. 808-813, June 2005.

[15] S. Bittner, W.U. Raffel, and M. Scholz, "The Area Graph-Based Mobility Model and Its Impact on Data Dissemination," Proc. Third IEEE Int'l Conf. Pervasive Computing and Comm. Workshops (PerCom), vol. 1, pp. 268-272, Mar. 2005.

[16] A.K.H. Souley and S. Cherkaoui, "Advanced Mobility Models for Ad Hoc Network Simulations," Proc. IEEE Systems Comm. (ICW), vol. 1, pp. 50-55, Aug. 2005.

[17] F. Legendre, V. Borrel, M.D.D. Amorim, and S. Fdida, "Reconsidering Microscopic Mobility Modeling for Self-Organizing Networks," IEEE Network, vol. 20, no. 6, pp. 4-12, Nov./Dec. 2006.

[18] T. Liu, P. Bahl, and I. Chlamtac, "Mobility Modeling, Location Tracking, and Trajectory Prediction in Wireless ATM Networks," IEEE J. Selected Areas in Comm., vol. 16, no. 6, pp. 922-936, Aug 1998.

[19] B.L. Mark and Z.R. Zaidi, "Robust Mobility Tracking for Cellular Networks," Proc. IEEE Int'l Conf. Comm. (ICC), vol. 1, pp. 445-449, May 2002.

[20] M. Hellebrandt and R. Mathar, "Location Tracking of Mobiles in Cellular Radio Networks," IEEE Trans. Vehicular Technology, vol. 48, no. 5, pp. 1558-1562, Sept. 1999.

[21] Z. Yang and X. Wang, "Joint Mobility Tracking and Hard Handoff in Cellular Networks via Sequential Monte Carlo Filtering," Proc. IEEE INFOCOM, vol. 2, pp. 968-975, June 2002. 
[22] L. Mihaylova, D. Angelova, S. Honary, D.R. Bull, C.N. Canagarajah, and B. Ristic, "Mobility Tracking in Cellular Networks Using Particle Filtering," IEEE Trans. Wireless Comm., vol. 6, no. 10, pp. 3589-3599, Oct. 2007.

[23] Z.R. Zaidi and B.L. Mark, "Mobility Estimation for Wireless Networks Based on an Autoregressive Model," Proc. IEEE Global Telecomm. Conf. (Globecom), vol. 6, pp. 3405-3409, Nov./Dec. 2004.

[24] R.G. Brown and P.Y. Hwang, Introduction to Random Signals and Applied Kalman Filtering, third ed. John Wiley \& Sons, 1997.

[25] J.S. Lim and A.V. Oppenheim, Advanced Topics in Signal Processing. Prentice Hall, 1987.

[26] J. Kempf, J. Wood, and G. Fu, "Fast Mobile IPv6 Handover Packet Loss Performance," Proc. IEEE Wireless Comm. and Networking Conf. (WCNC), vol. 2, pp. 1230-1235, Mar. 2003.

[27] Y. Gwon, G. Fu, and R. Jain, "Fast Handoffs in Wireless LAN Networks Using Mobile Initiated Tunneling Handoff Protocol for IPv4 (MITHv4)," Proc. IEEE Wireless Comm. and Networking Conf. (WCNC), pp. 1248-1252, Mar. 2003.

[28] Z.R. Zaidi and B.L. Mark, "A Mobility-Aware Handoff Trigger Scheme for Seamless Connectivity in Cellular Networks," Proc. IEEE 60th Vehicular Technology Conf. (VTC Fall), vol. 5, pp. 34713475, Sept. 2004.

[29] X. Hong, M. Gerla, G. Pei, and C.C. Chiang, "A Group Mobility Model for Ad Hoc Wireless Networks," Proc. ACM Int'l Conf. Modeling, Analysis and Simulation of Wireless and Mobile Systems (MSWiM), pp. 53-60, Aug. 1999.

[30] D. Lam, D.C. Cox, and J. Widom, "Teletraffic Modeling for Personal Communication Services," IEEE Comm. Magazine, vol. 35, no. 2, pp. 79-87, Feb. 1997.

[31] T. Tugcu and C. Ersoy, "Application of a Realistic Mobility Model to Call Admission in DS-CDMA Cellular Systems," Proc. IEEE Vehicular Technology Conf. (VTC), pp. 1047-1051, 2001.

[32] J. Scourias and T. Kunz, "An Activity-Based Mobility Model and Location Management Simulation Frameworks," Proc. ACM Int'l Conf. Modeling, Analysis and Simulation of Wireless and Mobile Systems (MSWiM), pp. 61-68, Aug. 1999.

[33] Z.R. Zaidi and B.L. Mark, "Real-Time Mobility Tracking Algorithms for Cellular Networks Based on Kalman Filtering," IEEE Trans. Mobile Computing, vol. 4, no. 2, pp. 195-208, Mar./Apr. 2005.

[34] J.J. Caffery Jr., Wireless Location in CDMA Cellular Radio Systems. Kluwer Academic Publishers, 1999.

[35] P. Deng and P.Z. Fan, "An AOA Assisted TOA Positioning System," Proc. Int'l Conf. Comm. Technology (WCC-ICCT), vol. 2, pp. 1501-1504, 2000.

[36] L. Cong and W. Zhuang, "Hybrid TDOA/AOA Mobile User Location for Wideband CDMA Cellular Systems," IEEE Trans. Wireless Comm., vol. 1, no. 3, pp. 439-447, July 2002.

[37] Y. Jeong, H. You, W.C. Lee, D. Hong, D.H. Youn, and C. Lee, "A Wireless Position Location System Using Forward Pilot Signal," Proc. IEEE Vehicular Technology Conf. (VTC), pp. 1354-1357, 2000.

[38] A. Abrardo, G. Benelli, C. Maraffon, and A. Toccafondi, "Performance of TDOA-Based Radiolocation Techniques in CDMA Urban Environments," Proc. IEEE Int'l Conf. Comm. (ICC), pp. 431-435, May 2002.

[39] G. Yost and S. Panchapakesan, "Automatic Location Identification Using a Hybrid Technique," Proc. IEEE Vehicular Technology Conf. (VTC), pp. 264-267, 1998.

[40] M. Gudmundson, "Correlation Model for Shadowing Fading in Mobile Radio Systems," Electronics Letters, vol. 27, pp. 2145-2146, Nov. 1991.

[41] G.L. Stüber, Principles of Mobile Communication, second ed. Kluwer Academic, 2001.

[42] D. Hong and S.S. Rappaport, "Traffic Model and Performance Analysis for Cellular Mobile Radio Telephone Systems with Prioritized and Nonprioritized Handoff Procedures," IEEE Trans. Vehicular Technology, vol. 35, no. 3, pp. 77-92, Aug. 1986.

[43] H.C. So and E.M.K. Shiu, "Performance of TOA-AOA Hybrid Mobile Location," IEICE Trans. Fundamentals, vol. E86-A, pp. 21362138, Aug. 2003.

[44] A.P. Dempster, N.M. Laird, and D.B. Rubin, "Maximum Likelihood from Incomplete Data via the EM Algorithm," J. Royal Statistical Soc. Series B, vol. 39, no. 1, pp. 1-38, 1977.

[45] C.F.J. Wu, "On the Convergence Properties of the EM Algorithm," The Annals of Statistics, vol. 11, no. 1, pp. 95-103, 1983.

[46] W. Mendenhall and T. Sincich, Statistics for the Engineering and Computer Sciences. Dellen, 1988.
[47] R.A. Johnson, D.W. Wichern, and D.W Wichren, Applied Multivariate Statistical Analysis. Prentice Hall, 2002.

[48] J. Broch, D.A. Maltz, D.B. Johnson, Y.C. Hu, and J. Jetcheva, "A Performance Comparison of Multi-Hop Wireless Ad Hoc Network Routing Protocols," Proc. ACM MobiCom, pp. 85-97, Oct. 1998.

[49] Z.R. Zaidi and B.L. Mark, "A Mobility Tracking Model for Wireless Ad Hoc Networks," Proc. IEEE Wireless Comm. and Networking Conf. (WCNC), vol. 3, pp. 1790-1795, Mar. 2003.

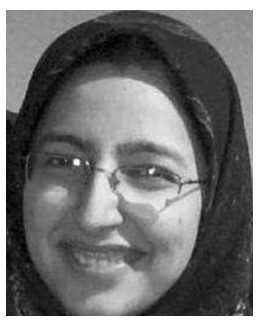

Zainab R. Zaidi received the BS degree in electrical engineering from the NED University of Engineering \& Technology, Karachi, Pakistan, in 1997, and the MS degree in electrical engineering and the PhD degree in electrical and computer engineering from George Mason University in 1999 and 2004, respectively. Currently, she is a researcher in the Networked Systems group of NICTA. Before joining NICTA in 2006, she taught at the NED University of Engineering \& Technology, Karachi, Pakistan, in 2005, and worked as a postdoctoral fellow in the Network Architecture and Performance Lab at George Mason University in 2004. Her research interests include different network layer issues in wireless mesh networks, such as robust routing, efficiency of link metrics, quality of service, etc., in addition to mobility tracking and its applications in mobile wireless networks. She is a member of the IEEE.

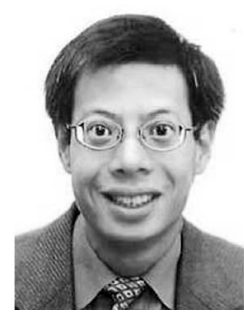

Brian L. Mark received the BASc degree in computer engineering with an option in mathematics from the University of Waterloo, Canada, in 1991 and the PhD degree in electrical engineering from Princeton University in 1995. He was a Research Staff member at NEC Labs from 1995 to 1999. In 1999, he was on part-time leave from NEC as a visiting researcher at Télécom ParisTech in Paris, France. In 2000, he joined the Department of Electrical and Computer Engineering at George Mason University, where he is currently a professor. His research interests lie in the design, modeling, and analysis of communication systems, computer systems, and communication networks. He is a senior member of the IEEE.

For more information on this or any other computing topic, please visit our Digital Library at www.computer.org/publications/dlib. 